Recepción: Mayo 05 de 2016

Aceptación: Junio 09 de 2016

Publicación: Septiembre 01 de 2016

\title{
Desarrollo y evaluación de simuladores virtuales para la enseñanza de competencias en el campo de la salud
}

Development and evaluation of virtual simulators for the teaching of competences in the field of health

\section{Georgina Cárdenas López}

Universidad Nacional Autónoma de México

Betzabé Sánchez

Universidad Nacional Autónoma de México

Emmanuel Castillo

Universidad Nacional Autónoma de México 


\section{Resumen}

Una desventaja a la que se enfrentan los estudiantes, durante su formación profesional, es la carencia de espacios y oportunidades en las cuales ponga en práctica sus conocimientos. La enseñanza de habilidades clínicas, al principio de la formación, por lo general implica el uso de libros de texto y de formatos clínicos en clase. Una posible solución a este problema, es llevar a cabo la formación en entornos clínicos reales, sin embargo, debido a las restricciones de tiempo, el costo de la supervisión, el acceso ineficaz a los escenarios reales y el error potencial; la formación del egresado para adquirir competencias clínicas profesionales se ve impactada de manera negativa. Para responder a esta carencia de práctica con la cual reducir las tasas de error de estudiantes y profesionales novatos en el campo de la salud mental, el Laboratorio de Enseñanza virtual y Ciberpsicología de la Facultad de Psicología de la UNAM, diseñó, desarrolló y evaluó una serie de cuatro simuladores virtuales de baja fidelidad para la enseñanza de habilidades técnicas y competencias profesionales, que replican las situaciones que ocurren en un escenario profesional, pero con la ventaja de que es posible controlar los eventos que ocurren en el ambiente virtual del simulador y recibir retroalimentación inmediata. El presente trabajo describe primeramente los resultados de la evaluación de usabilidad de la versión Beta de cuatro simuladores para la enseñanza de competencias clínicas en estudiantes de Psicología de la Universidad Nacional Autónoma de México: (1) entrevista Cognitivo Conductual, (2) entrevista motivacional, (3) entrevista conductual y (4) entrevista de recursos de afrontamiento. Así mismo, se informa de la evaluación de las estrategias de aprendizaje como son el modelamiento, moldeamiento y encadenamiento hacia atrás para el desarrollo de estrategias de aprendizaje simuladas. Los resultados obtenidos señalan que los simuladores con pacientes virtuales proporcionan a los estudiantes la oportunidad de mejorar los conocimientos, facilitar la adquisición de habilidades y competencias, así como su utilidad para disminuir la ansiedad y promover el juicio clínico en un ambiente seguro. La simulación resulta un instrumento de enseñanza poderoso para ayudar a los profesionistas a alcanzar los niveles más altos de competencia y por ende importante continuar desarrollando y evaluando desarrollos futuros.

\section{Abstract}

A disadvantage faced by students during their professional training is the lack of spaces and opportunities in which to put their knowledge into practice. Teaching clinical skills, at the beginning of training, usually involves the use of textbooks and clinical formats in class. One possible solution to this problem is to conduct 
training in real clinical settings, however due to time constraints, cost of supervision, ineffective access to real scenarios and potential error; The training of graduates to acquire professional clinical skills is negatively impacted. To respond to this lack of practice with which to reduce the error rates of students and new professionals in the field of mental health, the Laboratory of Virtual Teaching and Cyberpsychology of the Faculty of Psychology of the UNAM, designed, developed and evaluated a Series of four low fidelity virtual simulators for the teaching of technical skills and professional skills, which replicate the situations that occur in a professional setting, but with the advantage that it is possible to control the events that occur in the virtual environment of the simulator and receive Immediate feedback. The present work describes first the results of the usability evaluation of the Beta version of four simulators for teaching clinical competences in Psychology students of the National Autonomous University of Mexico: (1) Cognitive behavioral interview, (2) motivational interview, (3) behavioral interview, and (4) interviewing coping resources. Likewise, the evaluation of learning strategies such as modeling, shaping and backward chaining for the development of simulated learning strategies is reported. The results obtained indicate that simulators with virtual patients provide students with the opportunity to improve knowledge, facilitate the acquisition of skills and competences, as well as their usefulness in reducing anxiety and promoting clinical judgment in a safe environment. Simulation is a powerful teaching tool to help professionals reach the highest levels of competence and therefore important to continue to develop and evaluate future developments.

\section{Palabras Clave}

Simuladores virtuales, enseñanza, competencias, entrevista clínica, evaluación, salud.

\section{Keywords}

Virtual simulators, teaching competencies, clinical interview, evaluation, health.

\section{Introducción}

Existe una demanda importante por parte de estudiantes y profesionistas en el área de la salud para contar con una formación profesional que les garantice mayor eficiencia al incidir en la solución de problemas de salud mental. Para responder a tales situaciones, se requiere de personal que cuente con competencias profesionales para el diagnóstico, la intervención y evaluación de los servicios de salud que puedan ensayar y aplicar. Estas capacidades, se generan por medio de una formación que incluye el dominio de las técnicas y procedimientos de intervención efectivos. 
La medicina y la psicología entre otras profesiones de atención en el sector salud, tradicionalmente ha confiado en un "vea un, haga un" acercamiento al aprendizaje y experiencia. Esto inevitablemente expone a los pacientes a médicos y psicólogos inexpertos, por lo que los peligros y el daño asociado con esto son cada vez más inaceptables.

Para incidir en la solución de esta alternativa se propone que los profesionales que brindarán servicios de salud mental, cuenten con las competencias profesionales para el diagnóstico, la intervención y evaluación; dichas capacidades se pueden obtener por medio de una formación basada en competencias que incluye el dominio de las técnicas y procedimientos de intervención efectivos.

Para cubrir esta carencia de práctica y reducir los errores de profesionales en el campo de la salud, en concreto que se cometen con pacientes reales, se ha empleado con resultados positivos la llamada "simulación", siendo esta una réplica de las situaciones que ocurren en un escenario profesional, pero con la ventaja de que es posible controlar los eventos que ocurren. Es así, que el estudiante puede practicar las ocasiones necesarias en escenarios virtuales, contando además con la libertad de cometer errores, aprender de ellos y así alcanzar el dominio de la competencia clínica.

La simulación es una técnica que permite sustituir o amplificar experiencias reales de pacientes con experiencias dirigidas, artificialmente planeadas, que evoca o reproduce los aspectos sustanciales del mundo real en una manera totalmente interactiva. Como una estrategia educativa, la simulación proporciona la oportunidad de aprendizaje que es tanto inmersiva como empírica. $\mathrm{El}$ creciente empleo de tecnologías avanzadas para mejorar simuladores de enseñanza que favorezcan la seguridad del paciente, ha derivado en que en el área de asistencia médica usen la simulación en muchas formas, incluyendo a: pacientes simulados y virtuales, simuladores de maniquíes estáticos e interactivos, entrenadores de habilidades específicas, simulaciones con base en computadora y videojuegos "serios".

El principal uso de la simulación en el dominio de competencias profesionales es que los estudiantes ensayen las habilidades requeridas desarrollando un simulador que les permita la práctica deliberada. Como tal, ellos pueden cometer errores en un ambiente seguro, aprender de esos errores y alcanzar la habilidad logrando puntos de referencia predefinidos.

Entre otros, Navarro y Santillán (2009), señalan que los simuladores permiten la construcción de escenarios ideales para dotar al aprendiz de un recurso didáctico y esto se ve fundamentado en sus tres principales características: 1) Su papel motivacional debido a que permite la representación de fenómenos de estudio los cuales captan la atención del estudiante, 2) Cumple como facilitador de aprendizaje ya que el estudiante interactúa con el simulador favoreciendo la aprehensión de conocimientos a través del descubrimiento y 3) 
Su papel reforzador ya que permite al usuario la aplicación de los conocimientos adquiridos.

De esta manera, para mejorar la educación y en última instancia realzan la seguridad paciente, los profesionales de asistencia médica usan la simulación en muchas formas incluyendo a pacientes simulados y virtuales, simuladores de maniquíes estáticos e interactivos, entrenadores de tarea, simulaciones a base de pantalla (computadora) y juego "serio". Además, la simulación tiene el potencial para recrear escenarios que raras veces son experimentados y prueban a profesionales en situaciones desafiantes, y con cuidado volver a jugar o examinar su acciones. Esto es un instrumento de estudio poderoso para ayudar a profesionistas de la asistencia médica moderna a alcanzar los niveles más altos de competencia y mayor cuidado.

Más allá del impacto sobre el individuo y el trabajo del equipo, las técnicas de simulación proporcionan una oportunidad de mejorar el funcionamiento del sistema y son una clave importante para reducir los errores en la asistencia en salud. La investigación ha mostrado que las mejoras en el sistema pueden reducir las tasas de error y mejorar la calidad de la asistencia. Hay tres amplios dominios en los cuales la simulación es usada por profesionales de la asistencia médica. Primero, las técnicas de simulación pueden ser usadas para la práctica y la evaluación de procedimientos. Esto puede tomar una gran variedad de formas desde modelos de bancos simples a sofisticadas máquinas de realidad virtual. Segundo, los pacientes simulados o estandarizados tienen mucho tiempo que han sido usados para enseñar habilidades clínicas y son la base para la evaluación basada en el desempeño. Tercero, las tecnologías de simulación han sido usadas para el entrenamiento de equipos, el mejoramiento de la función en situaciones complejas llenas de tensión.

Además, existe evidencia de la relación entre el nivel de experiencia y la fidelidad del simulador. Un profesional de la salud principiante obtendrá más ganancia de un simulador de baja fidelidad que tiene la capacidad de enseñar habilidades genéricas. Un profesional experimentado requerirá el refinamiento de tareas, que con mayor probabilidad es obtenida de un simulador de fidelidad más alta que puede simular interacciones de tejido complejas o escenarios de crisis como el desangramiento o un episodio psicótico.

Desde esta perspectiva, este trabajo tuvo como objetivo principal el diseño, desarrollo la puesta a prueba de simuladores virtuales para la enseñanza de habilidades técnicas y competencias profesionales dirigidas a estudiantes y profesionistas en el campo de la salud mental. Se pretende que estos programas de enseñanza sea tanto eficaz (v.g., que logre una reducción de la tasa de error) como eficiente (v.g., amigables y aceptado por los profesionales de la salud).

Para ello, fue necesario desarrollar y poner a prueba la eficacia y eficiencia de nueve simuladores de realidad virtual para la adquisición de competencias profesionales en el ámbito clínico hospitalario, por lo que en el ámbito tecnológico se diseñaron herramientas novedosas basadas en realidad virtual inmersiva y 
aumentada que contenían estrategias instruccionales para el desarrollo de competencias profesionales en el campo de la salud.

Con el propósito de poner a prueba la efectividad de los simuladores virtuales, en términos de estrategia instruccional, se establecieron tres técnicas de aprendizaje para ser evaluadas y determinar los mejores resultados para la adquisición de competencias profesionales en el ámbito clínico. Considerando las tres técnicas más empleadas en simuladores desarrollados en medicina para el aprendizaje de una nueva conducta (Gallagher \& Satava, 2002), el simulador de entrevista conductual fue desarrollada con tres principios de aprendizaje: moldeamiento, encadenamiento inverso y desvanecimiento.

\section{Antecedentes}

\section{El empleo de la simulación en la enseñanza superior}

En la enseñanza superior, el uso de una técnica de simulación permite a los estudiantes tomar una decisión y luego observar el efecto de esta decisión en una amplia gama de situaciones de enseñanza como la medicina (Bidwell, CollinsNakai, Taylor, \& Jensen, 1985; Jones \& Keith, 1983; Melnick, 1990), ingeniería (Stevens \& Roberts, 1983), la física (Denis, Huynen, Lebrun, Martegani, \& Minas, 1981), biología (Wedekind, 1981) y la psicología (Benedict, Butts, \& Madison, 1981; Chan, Berven, \& Lam, 1990; Lambert \& Billings, 1991).

En el ámbito de la enseñanza de la psicología, las simulaciones han permitido a los estudiantes experimentar y explorar fenómenos complejos (Anderson, 1982; Bradley, 1989; Eamon, 1982; Lambert, Hedlund, \& Vieweg , 1990a; Lambert, Hedlund, \& Vieweg, 1990b; Petty \& Rosen, 1990). Por ejemplo, un programa de simulación ha brindado a los estudiantes la experiencia práctica en el uso de la técnica conductual de desvanecimiento para establecer un nuevo comportamiento, reforzando las aproximaciones más cercanas a la respuesta meta (Martin \& Pear, 1992). Los programas de simulación no sólo son utilizados para ilustrar los principios y fenómenos psicológicos, sino que también se han utilizado para enseñar diversas habilidades clínicas: (1) entrevista clínica a clientes (Hummel, Lichtenberg,\& Shaffer, 1975), (2) evaluación de víctimas de abuso infantil (MacFadden, 1989) y (3) programas de simulación que enseñan habilidades clínicas complejas, esto es, que implican decisiones de diagnóstico y tratamiento (Cárdenas, De la Rosa, Jurado \& Gutiérrez, 2012; García, Pericot, Gutiérrez, Ferrer \& Secades, 2012).

El trabajo en el campo de la salud requiere que los especialistas, que inciden en la vida de sus "pacientes", por lo que resulta indispensable cuenten con las competencias profesionales para el diagnóstico y la intervención que ofrecen (Flores, et al., 2010). Por ende, existe una demanda importante por parte 
de estudiantes y profesionistas para contar con una formación que incluya el dominio de estas técnicas y procedimientos de intervención (Moreno \& Cárdenas, 2012). El uso de la simulación como medio para crear espacios y situaciones de práctica para el entrenamiento de profesionales ha sido exitosamente aplicado en el área de la salud (Corvetto, et al., 2013).

En México, el Laboratorio para la enseñanza virtual de la Psicología de la UNAM, desarrolla desde el año 2001 de su fundación, desarrollos tecnológicos basadas en tecnologías de realidad virtual para el tratamiento de fobias y trastornos de ansiedad y cuenta sistemas tutoriales que incorporan hipertexto, simulación virtual y video digital para la enseñanza de competencias profesionales enfocadas al diagnóstico y planeación de la intervención psicológica para siete trastornos de ansiedad: ansiedad generalizada, fobia social, fobia específica, agorafobia, trastorno de pánico, trastorno obsesivo-compulsivo y estrés posttraumático. La evaluación controlada de estos sistemas resultó ampliamente satisfactoria.

Con base en la experiencia descrita previamente que el propósito de este proyecto se enfoca al diseño y desarrollo de simuladores virtuales dirigidos a mejorar las habilidades de profesionistas y estudiantes en el área de la salud, dirigidos a la enseñanza de competencias profesionales para conducir una entrevista clínica, el diagnóstico diferencial de trastornos psicológicos; así como para intervención en crisis en unidades de cuidados intensivos en los hospitales.

\section{Desarrollo de la propuesta}

\section{La estructura de los simuladores.}

El simulador virtual inicia con una breve introducción teórica sobre los componentes y características de cada tipo de entrevista, antes de dar comienzo a la práctica simulada; con el fin de mejorar el proceso de formación que permita al usuario iniciar la práctica simulada con el mismo nivel de conocimientos.

La estructura teórica, y guiones para la simulación de cada tipo de entrevista fueron supervisados, revisados y aprobados por expertos en contenidos sobre el tema. Los guiones desarrollados para cada uno de los simuladores; incluyen una entrevista realizada a partir de casos de pacientes reales, cuya situación fue adaptada a la pauta diseñada y contó con la asesoría de clínicos expertos acorde al tipo de entrevista. Para la simulación, se emplearon algunos de los problemas clínicos más comunes a cada tipo de entrevista, por ejemplo; en la entrevista conductual, las conductas obsesivas, fobias, entre otros.

Se diseñó y desarrolló una serie de simuladores virtuales para la enseñanza y el dominio de habilidades profesionales en diversos tipos de entrevista utilizados normalmente en la labor del psicólogo, como lo son la 
entrevista motivacional, la conductual, la cognitivo-conductual, entre otros. Dentro del simulador los usuarios cursan una introducción, en la cual repasan los conceptos básicos e importantes sobre el tipo de entrevista; el contenido que se revisa prescinde en gran medida el texto plano, sustituyéndolo por videos o imágenes que, además, sirven para modelar los ejemplos dados. Una vez que se concluye este primer apartado, se realiza una evaluación con el fin de determinar si efectivamente la persona ha obtenido el conocimiento teórico que servirá de base para su práctica. Cuando el usuario recibe una calificación satisfactoria, se le asigna un "paciente" virtual, con quien tendrá la oportunidad de realizar su práctica de entrevista. Lo que se espera es que cometa todos los errores en este ambiente controlado y no durante una situación real donde un error puede incidir negativamente en la vida de una persona. Durante la práctica se le ofrece retroalimentación, se evalúa su desempeño y se da contingencia a los errores cometidos para su corrección en intentos posteriores.

La estructura teórica, y guiones para la simulación de cada tipo de entrevista fueron supervisados, revisados y aprobados por expertos en contenidos sobre el tema. Los simuladores fueron desarrollados bajo el principio de aprendizaje de moldeamiento, los guiones desarrollados para cada uno de los simuladores incluyen una entrevista realizada a partir de casos de pacientes reales, cuya situación fue adaptada a la pauta diseñada y contó con la asesoría de clínicos expertos acorde al tipo de entrevista. Para la simulación, se emplearon algunos de los problemas clínicos más comunes a cada tipo de entrevista, por ejemplo; en la entrevista conductual, las conductas obsesivas, fobias, ansiedad y depresión; y para el programa de Afrontamiento Pro social, violencia en la pareja y adolescentes conflictivos.

Ilustración 1. Vista principal del Programa de Afrontamiento Pro Social

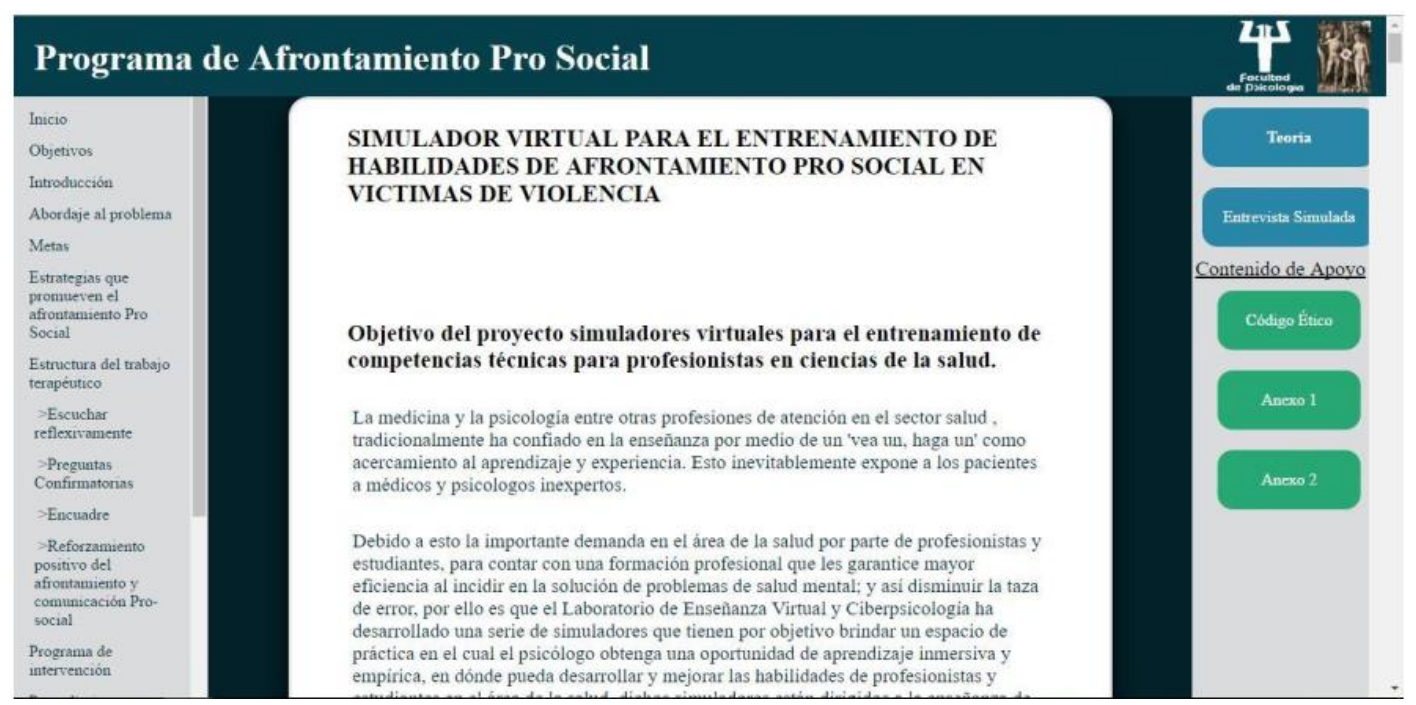

Fuente: Cárdenas, Sánchez \& Castillo (2016).

El simulador se lleva a cabo en primera persona, el usuario deberá tomar el papel de terapeuta en la entrevista y dirigirla. Se brinda al usuario una ficha de 


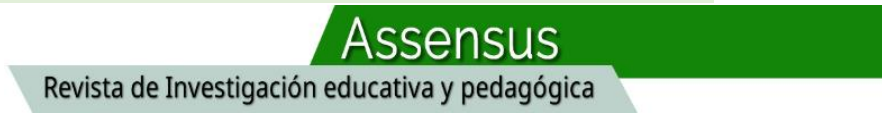

identificación y motivo de consulta; posteriormente el usuario toma el control de ahora en adelante, determinando que debe decir o preguntar en cada momento; se le ofrecerán opciones que puede elegir y de ser correctas el paciente las responderá y así la entrevista irá fluyendo, no obstante, en caso de ser incorrectas se ofrece retroalimentación al usuario.

Ilustración 2. Pantalla de Bienvenida del simulador

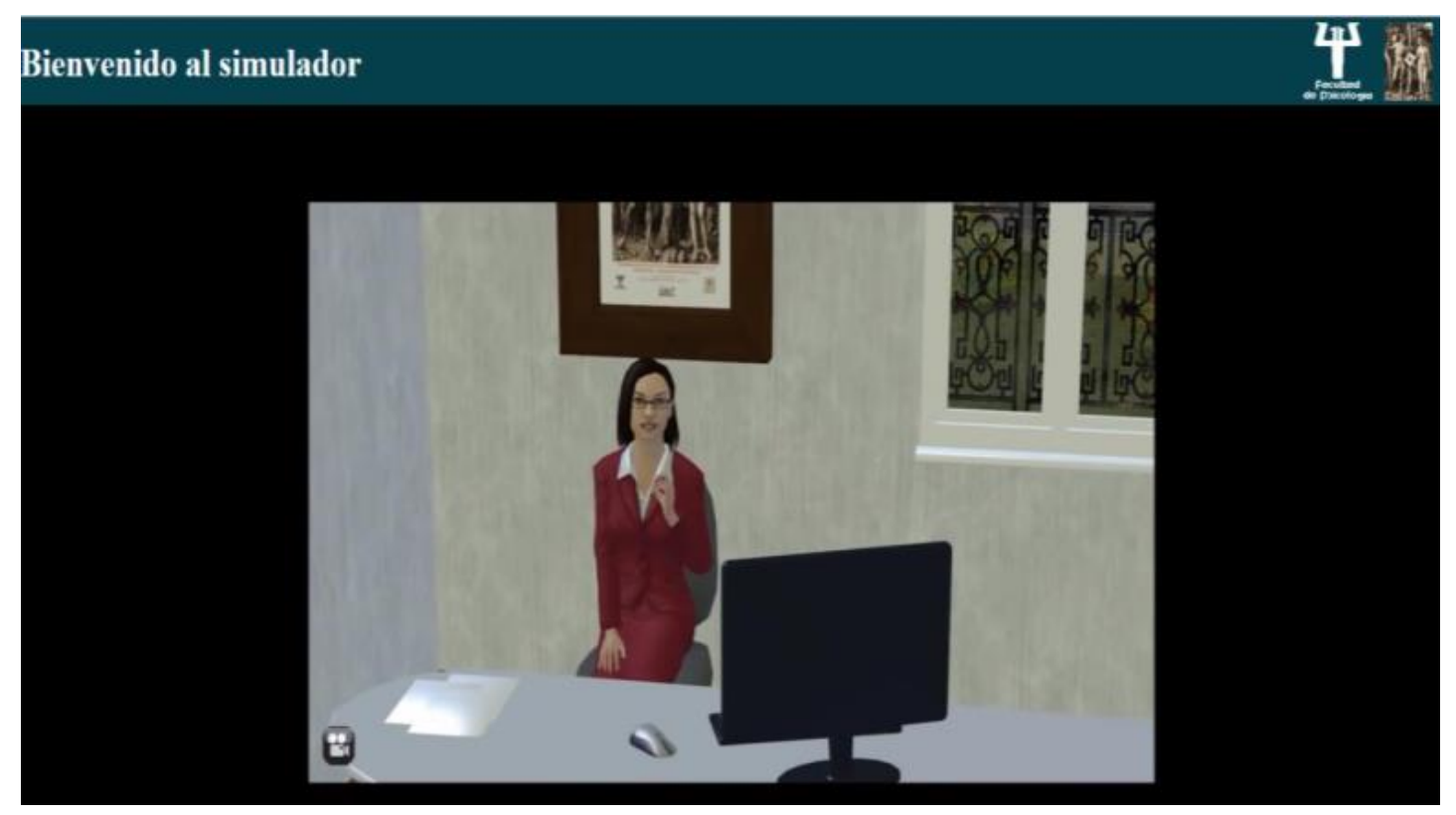

Fuente: Cárdenas, Sánchez \& Castillo (2016).

Ilustración 3. Simulación de entrevistas en el Programa de Afrontamiento Pro Social

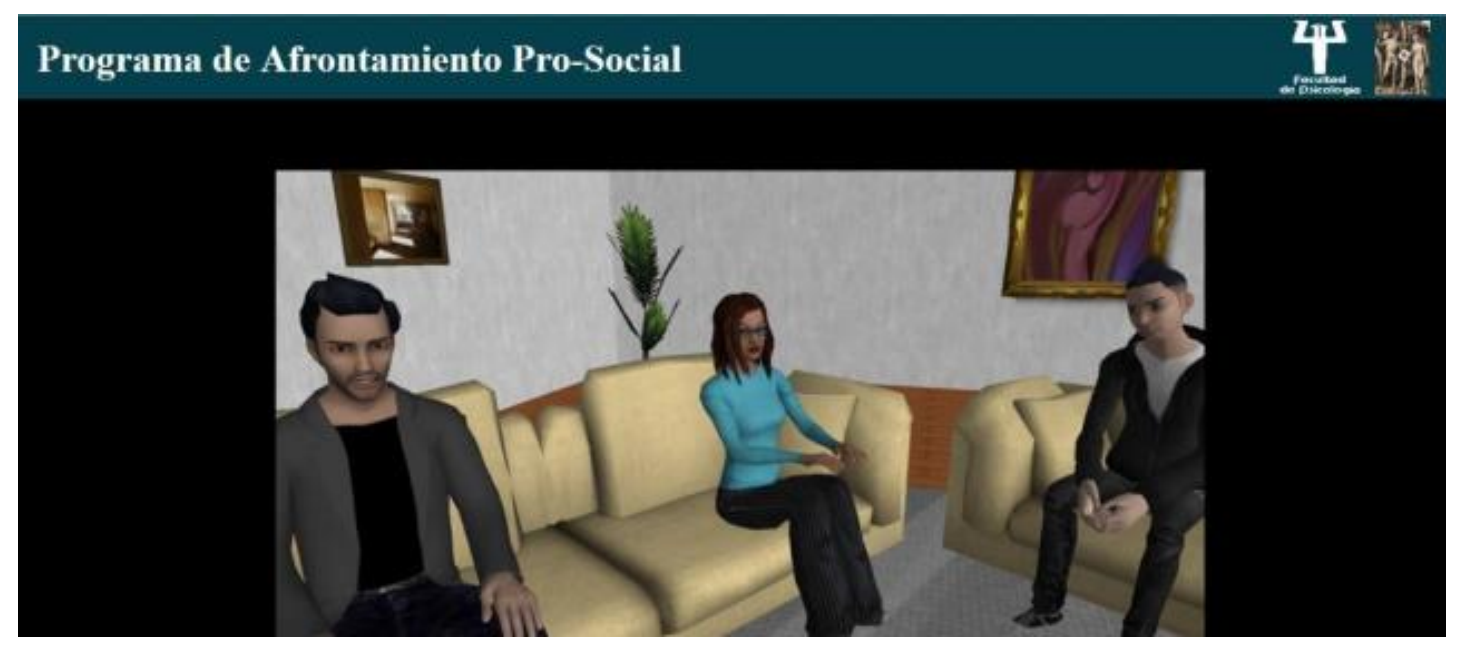

Fuente: Cárdenas, Sánchez \& Castillo (2016).

La dinámica que maneja el simulador en la plataforma para la práctica se lleva de la siguiente manera: se le brinda al usuario una breve descripción del paciente virtual (ficha de identificación y motivo de consulta), a continuación la pantalla mostrará la oficina en donde se llevará a cabo la entrevista; tras una breve animación en la cual el terapeuta invita a pasar al paciente y este se 
identifica, el usuario toma el control de ahora en adelante, determinando que debe decir o preguntar en cada momento; se le ofrecerán opciones que puede elegir y de ser correctas el paciente las responderá y así la entrevista irá fluyendo, no obstante, en caso de ser incorrectas se le ofrecerá una retroalimentación al usuario y qué parte de la sección teórica debe revisar para corregirlo.

\section{Simuladores virtuales para conducir entrevistas en psicología.}

Para el desarrollo de los simuladores virtuales de baja fidelidad para el entrenamiento de competencias profesionales para la entrevista y evaluación psicológica se eligieron por medio de una encuesta, el tipo de entrevistas más empleadas en el ámbito psicológico.

- Entrevista conductual (Llavona, Carrasco \& Carrasco, 1977; Muñoz, M. 1990; (Fernández \& Staats 1992).

- Entrevista motivacional (Rollnick, Miller, \& Butler, 2008).

- Entrevista clínica con enfoque cognitivo conductual (Fernández \& Staats, 1992).

- Evaluación del simulador.

\section{Metodología}

Se empleó un diseño aleatorio de tipo factorial entre grupos con 5 condiciones experimentales y un grupo control. Este diseño nos permitirá conocer la efectividad de las diversas modalidades de aprendizaje para la enseñanza de competencias con simuladores.

Este diseño asigna aleatoriamente a los participantes para recibir la intervención con diferentes herramientas de la siguiente manera:

$\mathrm{R} \mathrm{O}_{1} \mathrm{AO}_{2}$

$\mathrm{R} \mathrm{O}_{1} \mathrm{TAUO}_{2}$

Dónde:

$\mathrm{R}$ significa asignación aleatoria

$\mathrm{O}_{1}$ significa evaluación antes del tratamiento

$\mathrm{O}_{2}$ significa evaluación post-tratamiento

A significa aplicación del simulador virtual

TAU significa enseñanza tradicional

La muestra quedará conformada por un total de 60 participantes que serán asignados aleatoriamente a 2 grupos ( $N=30$ cada uno), 1 experimental y 1 control, 
bajo los lineamientos de un ensayo controlado aleatorizado con medidas de evaluación antes y después de la intervención para evaluar cada simulador virtual desarrollado.

\section{Variables Independientes.}

- Entrenamiento con simuladores para la enseñanza de habilidades técnicas y profesionales.

- Enseñanza "tradicional" (mira y hazlo).

\section{Variables Dependiente}

- Evaluación de conocimientos, tasas de error, satisfacción del usuario, correlación de los componentes del sistema con las competencias profesionales.

\section{Procedimiento}

La aplicación se llevó a cabo en un salón de la Facultad de Psicología de la UNAM. Para la evaluación del programa de Afrontamiento Pro Social, se utilizó una población de 42 estudiantes de tercer semestre para la evaluación teórica y entre ellos 4 estudiantes para la evaluación práctica, ninguno había tomado clases de entrevista ni contaban con experiencia previa en intervenciones psicológicas. Para la evaluación del simulador de entrevista conductual se realizó la evaluación con 7 voluntarios a quienes se aplicó la evaluación teórica y práctica.

Para la evaluación de habilidades prácticas se pidió a los participantes que realizaran una entrevista previa a la simulación con un paciente ficticio, la cual evaluó mediante una lista cotejable las habilidades del terapeuta antes de tener contacto con el simulador, así mismo se aplicó un instrumento previo acerca del contenido teórico del simulador correspondiente, una vez que se obtuvieron esas evaluaciones se presentó a los usuarios el simulador y se permitió que se usara libremente durante espacio de tres horas, al finalizar ellos debían volver a contestar el instrumento teórico del simulador, y posteriormente se les evaluó nuevamente de manera práctica mediante una simulación con un paciente ficticio, a estos instrumentos se sumaron instrumentos de usabilidad, funcionalidad y satisfacción, para conocer lo el grado de usabilidad del simulador, es decir el grado en que un sistema interactivo es fácil y agradable de usar. La usabilidad es un concepto fundamental para la interacción humano- computadora que nos permite evaluar y por medio de ello mejorar el producto final.

\section{Resultados}

\section{Simulador de Entrevista motivacional.}

El objetivo fue moldear la competencia final, que es llevar a cabo una entrevista completa sin errores. Con el propósito de evaluar la efectividad del 
programa de enseñanza por medio de simuladores virtuales, cuyo objetivo es el de apoyar en el desarrollo de las habilidades en se procedió a instrumentar y evaluar la efectividad de entrevista conductual, empleando un diseño pre-test, post-test para conocer sí el usuario había adquirido la competencia entrenada, esto es conducir una entrevista conductual.

Para su evaluación 10 participantes, estudiantes o egresados de la carrera de psicología, aceptaron participar para ensayar con el simulador virtual, de los cuales 5 participantes poseían experiencia previa realizando entrevistas en ambientes profesionales y otros 5 participantes sin experiencia previa que no fueran las clases que cursaron durante su carrera.

Se evaluaron los conocimientos sobre la entrevista conductual de cada uno antes y después practicar con el simulador para registrar si efectivamente hubo un cambio; así mismo, se les administro una serie de cuestionarios con el objetivo de medir la usabilidad del simulador de acuerdo a la experiencia de cada uno. Para llevar a cabo el estudio, cada participante ingresaba en una sala donde sólo había una computadora, un escritorio y una silla y se les dotaba de privacidad, pues el evaluador sólo entraba a la sala al principio y al final para dar instrucciones y aplicar los cuestionarios. El tiempo en que podían interactuar con el material, repasando los contenidos o practicando la entrevista fue libre, cada participante determinaba en que momento deseaba que le aplicaran la evaluación final. Se registró 45 minutos como la media del tiempo que le llevaba a cada uno completar la tarea. El cuestionario aplicado consta de 10 preguntas de opción múltiple, basadas $100 \%$ en el contenido presentado, en el cual cada pregunta tiene el valor de 1 punto, por lo que la calificación obtenida por el participante puede variar entre 0 y 10 puntos.

Los resultados obtenidos arrojan lo siguiente: La media obtenida por los participantes durante su evaluación inicial fue de 5.8; la media obtenida por los participantes en su evaluación posterior al entrenamiento fue de 8. Estos resultados confirman la hipótesis de que los participantes obtendrían mejores resultados durante su evaluación posterior y que los participantes con experiencia obtuvieron mejores resultados que aquellos que no contaban con experiencia.

En cuanto a la evaluación de usabilidad, los resultados obtenidos a partir de la Escala de Usabilidad de los Sistemas arrojaron lo siguiente: el Valor Global (VG) de usabilidad, con una media de 80.75 puntos, se considera superior a la media (70 puntos). Las respuestas registradas por los formatos de evaluación de satisfacción y funcionalidad, se discutirán, y serán utilizados con el fin de mejorar aspectos del diseño de los simuladores en esta etapa temprana de su desarrollo

\section{Simulador entrenamiento en estrategias de Afrontamiento PAPS.}

Tras las primeras evaluaciones realizadas a ambos simuladores, los resultados indican que existe un cambio positivo en el desempeño de los 
participantes, en ambos casos existe un aumento del puntaje obtenido en las evaluaciones posteriores al uso del simulador.

En el simulador de Afrontamiento Pro-social de 38 participantes que concluyeron el simulador del Programa PAPS, 24 de ellos elevaron su puntaje respecto a su primer evaluación. Como se puede observar en la tabla 1, ambos simuladores obtuvieron un aumento de aciertos en sus evaluaciones tras el uso del simulador.

Tabla 1. Comparación grupal entre la media de resultados obtenidos en el pretest con la media de resultados obtenidos en el postest y resultados de la media de usabilidad de ambos simuladores, PAPS $\mathrm{N}=38$ y Conductual $\mathrm{N}=8$

\begin{tabular}{llll}
\hline & $\begin{array}{l}\text { Media } \\
\text { PRETEST }\end{array}$ & $\begin{array}{l}\text { Media } \\
\text { POSTEST }\end{array}$ & $\begin{array}{l}\text { Media de } \\
\text { Usabilidad }\end{array}$ \\
\hline $\begin{array}{l}\text { Simulador } \\
\text { Entrevista }\end{array}$ & 4 & 7.5 & 7.5 \\
$\begin{array}{l}\text { Conductual } \\
\text { Simulador }\end{array}$ & 14.76 & 16.11 & 70.197 \\
PAPS & & & \\
\hline
\end{tabular}

Fuente: Cárdenas, Sánchez \& Castillo (2012).

En cuanto a la evaluación práctica que se realizó a los cuatro estudiantes que cursaron el simulador PAPS, también mostró cambios respecto a su desempeño antes de la interacción con el simulador, además los estudiantes comentaron sentirse más confiados en realizar la intervención ante un paciente real. Lo mismo sucedió con el simulador de entrevista conductual, los participantes obtuvieron una mejor evaluación de su simulación con paciente ficticio tras el uso del simulador. Los resultados del Instrumento Sistem Usability Scale, muestra que los simuladores tienen una interfaz sencilla y por lo tanto son usables, el simulador del Programa de afrontamiento Pro social obtuvo una media de 70.197, y el simulador de entrevista conductual una media de 75, puntos sobre la media de 68 (Sauro, 2011).

\section{Conclusiones}

Los resultados de usabilidad y satisfacción de los usuarios evaluados son satisfactorios para el entrenamiento de competencias profesionales en enseñanza superior y muestran concordancia con otros autores. Se espera continuar con este tipo de desarrollos que son de tanta importancia y apoyo para los nuevos terapeutas que se entrenan actualmente de la misma forma que se entrenaban hace la salud, ya es de vital importancia contar con las habilidades necesarias para atenderles de la mejor manera.

Con base en evaluaciones de usabilidad que se han realizado, la técnica de simulación virtual puede ser un medio potencialmente eficaz para la 
adquisición de competencias clínicas en la instrucción en psicología, por lo que es necesario fortalecer la base empírica con la que actualmente se cuenta, mediante estudios controlados que nos permitan realizar análisis de regresión lineal para la identificación de variables mediadoras que potencialicen la efectividad de los simuladores virtuales de baja fidelidad desarrollados. Así mismo, es importante destacar que la simulación tiene el potencial para recrear escenarios que raras veces son experimentados y prueban a profesionales en situaciones desafiantes, y con cuidado volver a jugar o examinar su acciones. Esto es un instrumento de estudio poderoso para ayudar a los profesionistas a alcanzar los niveles más altos de competencia y por ende importante explorar en desarrollos futuros.

Es necesario desarrollar novedosas interfaces de navegación e interacción adaptadas a los simuladores virtuales para la enseñanza, especialmente diseñados para México que considere los significados y contextos socioculturales. Se considera que los simuladores virtuales representan métodos de enseñanza que permitirán estrategias instruccionales que mejoren notablemente los actuales.

\section{Referencias Bibliográficas}

Anderson, D. E. (1982). Computer simulations in the psychology laboratory. Simulation \& Games,13. 13-36

Benedict, J. O., BUTTS,B.D., \&MADISON.J.(1981).Computer simulation or real experimentation: Is one better for teaching experimental design? Teaching of Psychology, 8, 35-38

Bidwell, C. M., COLLINS-NAKAI, R. L., TAYLOR, W. o., \& JENSEN, W. A. (1985). Multidisciplinary team production of computer-based simulations to teach pediatrics. Journal of Medical Education, 60. 397-403

Bradley, D. R. (1989). A general purpose simulation program for sta- tistics and research methods. Advances in Social Science \& Computers. I, 145-186

Cárdenas, G., Bouchard, S., \& Vite, A. (2015) Realidad Virtual: Aplicaciones en E-Salud. Universidad nacional autónoma de México. México.

Cárdenas, L.G.,De la Rosa A., Jurado S. \& Gutierrez, M. (2012) Evaluación de un simulador, diagnóstico. Simposio "Psicopedagogía en educación a distancia: investigación y práctica". Facultad de Psicología, México D.F, UNAM 22-24 de agosto del 2012.

Cárdenas, G., Solano, M., Olivares, E., y Tezcucano A., (2003), Adaptación del Manual de entrenamiento del Programa Afrontamiento Pro Social.

Denis, J. P., Huynen, A. M., Lebrun, M., Martegani, A., \& Mine, P. (1981). Simulation and learning by discovery. In D. Wil- denberg (Ed.), Computer simulation in university teaching (pp.35-41). Amsterdam: North-Holland.

Eamon, D. B. (1982). CEDATS: A cognitive experimental design and testing system. Behavior Research Methods \& Instrumentation, 14, 142-145 
Corvetto, Marcia, Bravo, María Pía, Montaña, Rodrigo, Utili, Franco, Escudero, Eliana, Boza, Camilo, Varas, Julián, \& Dagnino, Jorge. (2013). Simulación en educación médica: una sinopsis. Revista médica de Chile, 141(1), 70-79.

Fernández R. \& Staats (1992) Paradigmatic behavioral assessment: answering the crisis of behavioral assessment, Advances in Behaviour Research and Therapy,14(1),1-29

Flores Villalba, E., Díaz Elizondo, J., Leyva Alvizo, A., Villegas Cabello, O., Fernández Rangel, E. \& del Real Romo, Z. (2010) Simuladores virtuales laparoscópicos para el entrenamiento de cirujanos: una realidad. Revista Avances, 7(21), 41-44.

Gallagher, A.G., \& Satava, R.M. (2002) Virtual reality as a metric for the assessment of laparoscopic psychomotor skills. Learning curves and reliability measures. Surgical Endoscopy, 16(12), pp. 1746-1752

Hummel, T. J., Lichtenberg, J. W., \& Shaffer, W. F. (1975). CLIENT 1: A computer program which simulates client behavior in an initial interview. Journal of Counseling Psychology, 22,164-169

Jones. G. L. \& Keith, K. D. (1983). Computer clinical simulations in health sciences. Journal of Computer-Based Instruction,9. 108-114

Lambert, M. E., Hedlund, J.L., \& Vieweg, B. W. (1990a). Computer simulations in mental health education: Current status. Computers in Human Services, 7, 211-229.

Lambert, M. E., Hedlund, J.L., \& Vieweg, B. W. (1990b). Computer simulations in mental health education: Two illustrative projects. Computers in Human Services, 7, 231-245.

Macfadden, R. J. (1989). Sexual abuse assessment training: Devel- oping CAl in child welfare. Computers in Human Services, 5(3-4), 29-41

Melnick,D.E. (1990). Computer-based clinical simulations: State of the art. Evaluation in the health Professions. Vol 13. Database: PsyclNFO 104-120

Martin, G., \& Pear, J. J. (1992). Behavior modification: What it is and how to do it (4th ed.). Englewood Cliffs, NJ: Prentice-Hall

Moreno Almazán, O. \& Cárdenas López, M. G. (2012). Perfiles de Alumnos de Psicología en México. Revista Perfiles Educativos, 34(36), 118-136.

Muñoz, M. (1990) citado en Crespo, M. \& Larroy, C. (1998) Técnicas de modificación de conducta: guía práctica y ejercicios. Madrid: Dykinson.

Navarro, R. E., \& Santillán, A. G (2009). Un modelo didáctico basado en el diseño de simuladores: el.Ide@s CONCYTEG. Año 4, Núm. 46, $14 . \quad$ Recuperado de http://concyteg.gob.mx/ideasConcyteg/Archivos/46082009_MODELO_DIDACTICO_BASADO_EN _SIMULADORES.pdf

Petty, L. C., \& Rosen, E. F. (1990). Increase in mastery levels using a computer-based tutorial/simulation in experimental psychology. Behavior Research Methods, Instruments, \& Computers, 22, 216- 218.

Rollnick, S., Miller, W \& Butler, C.C. (2008). Motivational interviewing in health care. New York: The Guilford Press.

Sauro, J. (2011). Measuring usability with the system usability scale (SUS). http://www.measuringu.com/sus.php (02/12/15)

Stevens, A \& Roberts, B. (1983) Quantitative and Qualitative Simulation in Computer Based training Journal of Computer Based Instruction, vol 10. No. $1 \& 2$. 
Desarrollo y evaluación de simuladores virtuales para la enseñanza de competencias en el campo de la salud.

\section{Assensus}

Revista de Investigación educativa y pedagógica

Wedekind, J. P. (1981). The instructional use of computer simulation in the teaching of biology: Three examples. In D. Wildenberg (Ed.), Computer simulation in university teaching (pp. 223-235). Amsterdam: North- Holland 